\title{
Observation of the Chiral-Anomaly-Induced Negative Magnetoresistance in 3D Weyl Semimetal TaAs
}

\author{
Xiaochun Huang, ${ }^{1}$ Lingxiao Zhao, ${ }^{1}$ Yujia Long, ${ }^{1}$ Peipei Wang, ${ }^{1}$ Dong Chen, ${ }^{1}$ Zhanhai Yang, ${ }^{1}$ Hui Liang, \\ Mianqi Xue, ${ }^{1}$ Hongming Weng, ${ }^{1,2}$ Zhong Fang, ${ }^{1,2}$ Xi Dai, ${ }^{1,2}$ and Genfu Chen ${ }^{1,2, *}$ \\ ${ }^{1}$ Beijing National Laboratory for Condensed Matter Physics, and Institute of Physics, \\ Chinese Academy of Sciences, Beijing 100190, China \\ ${ }^{2}$ Collaborative Innovation Center of Quantum Matter, Beijing 100084, China
}

(Received 14 May 2015; published 24 August 2015)

\begin{abstract}
Weyl semimetal is the three-dimensional analog of graphene. According to quantum field theory, the appearance of Weyl points near the Fermi level will cause novel transport phenomena related to chiral anomaly. In the present paper, we report the experimental evidence for the long-anticipated negative magnetoresistance generated by the chiral anomaly in a newly predicted time-reversal-invariant Weyl semimetal material TaAs. Clear Shubnikov de Haas $(\mathrm{SdH})$ oscillations have been detected starting from a very weak magnetic field. Analysis of the SdH peaks gives the Berry phase accumulated along the cyclotron orbits as $\pi$, indicating the existence of Weyl points.
\end{abstract}

DOI: 10.1103/PhysRevX.5.031023

\section{INTRODUCTION}

When two nondegenerate bands cross in threedimensional (3D) momentum space, the crossing points are called Weyl points, which can be viewed as magnetic monopoles [1] or topological defects in the band structure, like "knots" on a rope. Near Weyl points, the low-energy physics can be described by Weyl equations [2] with distinct chirality (either left- or right-handed), which mimics relativistic field theory in particle physics. On a lattice system, Weyl points always appear in pairs with opposite chirality and are topologically stable against perturbations that keep translational symmetry [3-6]. If two Weyl points with opposite chirality meet in momentum space, they will generally annihilate each other, but they may also be stabilized as 3D Dirac points by additional (such as crystalline) symmetry [7-10]. For materials with Weyl points located near the Fermi level, called Weyl semimetals (WSMs), exotic low-energy physics can be expected, such as Fermi arcs on the surface [4,5] and the chiral-anomaly-induced quantum transport [11-14]. Recently, 3D Dirac semimetals, $\mathrm{Na}_{3} \mathrm{Bi}$ and $\mathrm{Cd}_{3} \mathrm{As}_{2}$, have been theoretically predicted $[8,9]$ and experimentally confirmed [15-19], while WSMs are still waiting for

\footnotetext{
*Corresponding author. gfchen@iphy.ac.cn

Published by the American Physical Society under the terms of the Creative Commons Attribution 3.0 License. Further distribution of this work must maintain attribution to the author(s) and the published article's title, journal citation, and DOI.
}

Subject Areas: Condensed Matter Physics,

Materials Science,

Topological Insulators experimental verification in spite of various theoretical proposals [4,5,20-24].

The anomalous dc transport properties are an important consequence of the topological band structure $[13,25,26]$. In topological insulators (TI), the transport properties are dominated by the topological surfaces states (SS), where the lack of backscattering caused by the unique spin structure of the SS leads to the weak antilocalization (WAL) behavior. However, in WSMs the bulk states are semimetallic and dominate the dc transport. In relativistic field theory, for a continuing system described by the Weyl equation, chiral anomaly can be understood as the nonconservation of the particle number with given chirality, which only happens in the presence of parallel magnetic and electric fields [11]. For any realistic lattice systems, the chiral anomaly then manifests itself in the intervalley pumping of the electrons between Weyl points with opposite chirality. In the noninteracting case, the chiral anomaly can be simply ascribed to the zeroth Landau levels, which are chiral and have opposite signs of the velocity for states around Weyl points with opposite chirality, as shown in Fig. 1(d) [11]. The additional electric field parallel to the magnetic field will then generate charge imbalance between two chiral nodes, leading to an electric current that can only be balanced by intervalley scattering. Considering the fact that for clean samples the intervalley scattering time is extremely long and the degeneracy of the Landau level is proportional to the magnetic field strength, the chiral anomaly in WSM will, in general, lead to negative magnetoresistance $(\mathrm{MR}=[\rho(H)-\rho(0) / \rho(0)])$ when the magnetic field is parallel to the current. On the other hand, for ordinary metal or semiconductors, the MR 
is weak, positive, and usually not very sensitive to the magnetic field direction. Therefore, the negative and highly anisotropic MR has been regarded as the most prominent signature in transport for the chiral anomaly, and it indicates the existence of 3D Weyl points. In addition, the chiral anomaly can also generate other fascinating phenomena, i.e., the anomalous Hall effect and the nonlocal transport properties $[5,12]$.

Using first-principle calculations, Weng et al. [23] predicted that a family of binary compounds represented by TaAs are time-reversal-invariant 3D WSMs with a dozen pairs of Weyl nodes that are generated by the absence of an inversion center. The exotic Fermi arch on the surface and Weyl nodes in the bulk have been identified by angleresolved photoemission spectroscopy and microwave transmission measurements [27-30]. Materials in the TaAs family are completely stoichiometric and nonmagnetic, providing an almost ideal platform for the study of the chiral anomaly in WSM. In this work, we perform transport studies of the TaAs single crystal down to $1.8 \mathrm{~K}$, with a magnetic field up to $9 \mathrm{~T}$. Ultrahigh mobility $\left(\mu_{e} \approx 1.8 \times 10^{5} \mathrm{~cm}^{2} \mathrm{~V}^{-1} \mathrm{~s}^{-1}\right.$ at $10 \mathrm{~K}$ ) has been found with a multiband character. Extremely large positive MR $(\approx 80000 \%$ at $1.8 \mathrm{~K}$ in a field of $9 \mathrm{~T})$ is discovered for a magnetic field perpendicular to the current (or the external electric field). Ultrahigh mobility and large MR in the same material have also been detected by Zhang et al. [31]. Unfortunately, in the configuration of $B / / I$, their MR data remain positive in the whole magnetic field up to $9 \mathrm{~T}$. In our work, when the magnetic field is rotated to be parallel to the current, notable negative MR has been observed, demonstrating the chiral anomaly effects in this particular material. Strong $\mathrm{SdH}$ oscillations have been found from a very low magnetic field, from which two sets of oscillation frequencies can be extracted, indicating two types of carriers, in good consistency with our firstprinciples calculations.

\section{CRYSTAL STRUCTURE AND MEASURING METHOD}

TaAs crystallizes in a body-centered-tetragonal NbAstype structure with a nonsymmorphic space group of $I 4_{1} m d$, in which the $c$ axis is perpendicular to the $a b$ plane [see Fig. 1(a)]. The lattice parameters are $a=b=$ $3.4348 \AA$ and $c=11.641 \AA$ [32]. Because of the lack of inversion symmetry, first-principles calculations predicted a dozen pairs of Weyl points in the Brillouin zone (BZ) [23]. A schematic diagram of theoretically predicted Weyl nodes projected on the (001) facet can be seen in Fig. 1(b). In this study, the single crystals of TaAs were grown by chemical vapor transport. A polycrystalline TaAs that previously reacted was filled in the quartz ampoule using $2 \mathrm{mg} / \mathrm{cm}^{3}$ of iodine as the transporting agent. After evacuating and sealing, the ampoule was kept at the growth (a)

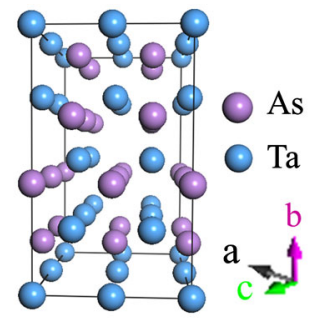

(b)
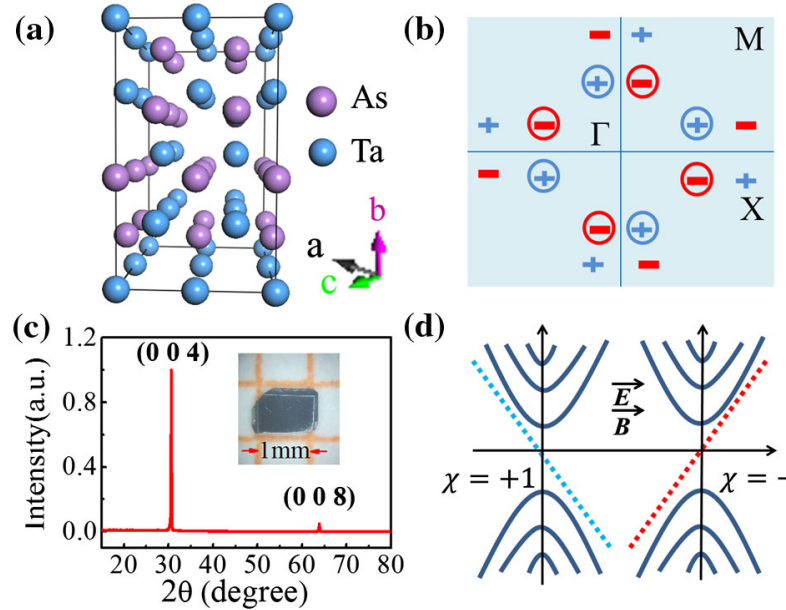

(d)

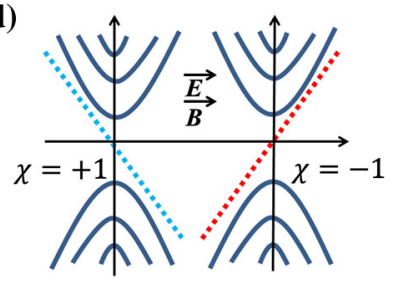

FIG. 1. Structure and symmetry of a TaAs single crystal. (a) The crystal structure of TaAs with a nonsymmorphic space group of $I 4_{1} \mathrm{md}$. Blue and violet balls represent a Ta atom and an As atom, respectively. (b) Schematic diagram of a dozen pairs of Weyl points projected on the (001) facet. "+" and "-" denote Weyl points with positive and negative chiralities, respectively. The circles show that there are two Weyl points with the same chirality projected on the same point in the (001) facet. $\Gamma, X$, and $\mathrm{M}$ are the high symmetry points in the Brillouin zone. (c) X-ray diffraction pattern of a TaAs single crystal. The inset shows an optical image of a typical sample at the millimeter scale. (d) Schematic diagram of bulk Landau levels of a pair of Weyl nodes. The dotted lines represent the zeroth quantum Landau Level with "+" (blue) and "-" (red) chiralities in a magnetic field parallel to the electric current.

temperature for three weeks. Large polyhedral crystals with dimensions up to $1.5 \mathrm{~mm}$ are obtained in a temperature field of $\Delta T=1150^{\circ} \mathrm{C}-1000^{\circ} \mathrm{C}$. Figure 1 (c) shows the $\mathrm{x}$-ray diffraction (XRD) from a TaAs crystal oriented with the scattering vector perpendicular to the (001) plane. The inset is the morphology of a representative crystal looking down the [001] direction. The crystal was polished into a rectangular sample $\left(1 \times 0.3 \times 0.08 \mathrm{~mm}^{3}\right)$ for magnetoresistance and Hall resistivity measurements using fourpoint probe and alternating current transport methods in the Quantum Design PPMS. The electric current is always applied parallel to the (001) plane along the $a$ or $b$ axis in our studies. For MR (or Hall resistivity) measurements, any additional Hall (or resistive) voltage signals due to the misalignment of the voltage leads have been corrected by reversing the direction of the magnetic field. Firstprinciples calculations are performed by using the OpenMX [33] software package. The choice of a pseudo-atomic orbital basis set with Ta9.0-s2p2d2f1 and As9.0-s2p2d1, the pseudopotential and the sampling of BZ $(10 \times 10 \times 10 k$ grid $)$ have been checked. The exchangecorrelation functional within a generalized gradient approximation parametrized by Perdew, Burke, and Ernzerhof has been used [34]. The optimized lattice 
constants $a=b=3.4824 \AA, c=11.8038 \AA$, and atomic sites are in agreement with the experimental values.

\section{RESULTS AND DISCUSSION}

\section{A. Magnetoresistance measurements}

Figure 2 presents the MR measured at $1.8 \mathrm{~K}$ by tilting the magnetic field $(B)$ at an angle $(\theta)$ with respect to the electric current $(I)$. The Hall signal has been removed by averaging the $\rho_{\mathrm{xx}}$ data over positive and negative field directions. As shown in Fig. 2(a), when the magnetic field is applied perpendicular to the current $\left(B \perp I, \theta=0^{\circ}\right)$, a surprising positive MR of up to $80000 \%$ is observed. Near zero field, MR exhibits quadratic field dependence, which soon changes to almost linear dependence at a very low field without any trend towards saturation up to $9 \mathrm{~T}$. This giant conventional MR strongly relies on $\theta$ and decreases considerably with increasing $\theta$. When the magnetic field is rotated parallel to the electric current $\left(\theta=90^{\circ}\right)$, we observe negative MR, strong evidence of Weyl fermions in TaAs. Elaborate measurements at different angles around $\theta=90^{\circ}$ are implemented and presented in Fig. 2(b). As shown in the main panel, by rotating $\theta$ from $87^{\circ}$ to $91.8^{\circ}$, negative MR arises in the cases of $\theta$ between $88^{\circ}$ and $91.5^{\circ}$, and it reaches a maximum $(-30 \%)$ at $\theta=90^{\circ}$ $(B / / I)$. This can also be intuitively viewed as a consequence of the steep downturn of MR in the magnetic field range $1 \mathrm{~T}<B<6 \mathrm{~T}$ (and $-1 \mathrm{~T}<B<-6 \mathrm{~T}$ ). In this range, for clarity, the minima of MR curves at different angles are listed in the inset of Fig. 2(b). The largest value, as expected, occurs at $\theta=90^{\circ}$. We note that the negative MR in Fig. 2(b) disappeared as we rotated the field about $2^{\circ}$ away from the current. This seems hard to believe. However, it makes sense when we recall that the conventional positive $\mathrm{MR}(B \perp I)$ of TaAs is very large $(\approx 80000 \%$ at $1.8 \mathrm{~K}$ in a field of $9 \mathrm{~T}$ ) and increases remarkably with an increasing magnetic field. So, a slightly imperfect alignment of the magnetic field and the current in the sample will arouse a large perpendicular component and obscure the negative MR, especially in a large field. Thus, the negative MR is confined to about $\pm 2^{\circ}$ of $B / / E$. In other words, in a system with smaller positive MR (at $\theta=0^{\circ}$ ), we may observe larger negative MR (at $\theta=90^{\circ}$ ) in a wide magnetic field range. Indeed, larger negative MR has been observed in $\mathrm{Na}_{3} \mathrm{Bi}$ [35] and $\mathrm{TaP}$ [36], which have much smaller positive MR than that of TaAs at $\theta=0^{\circ}$.

The origin of the negative MR in TaAs can be explained by the chiral anomaly in the semiclassical
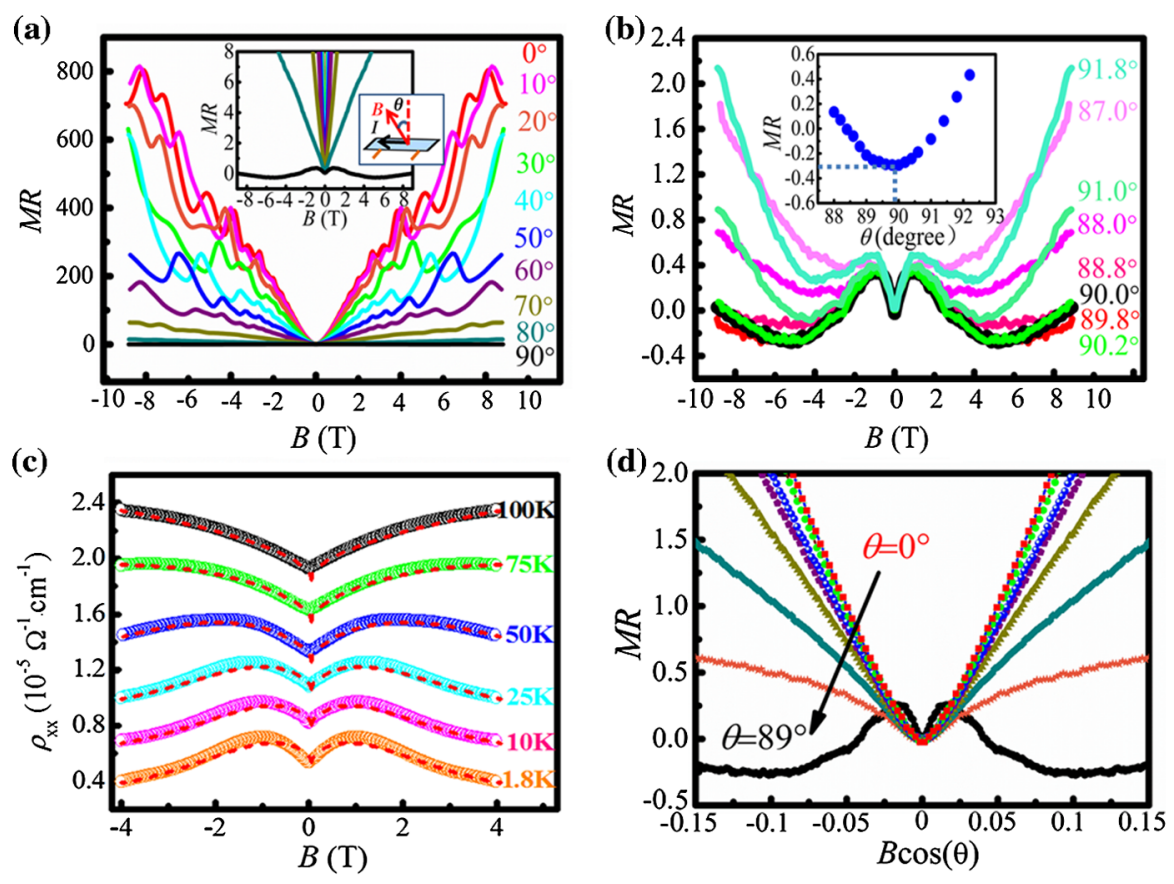

FIG. 2. Angular and field dependence of MR in a TaAs single crystal at $1.8 \mathrm{~K}$. (a) Magnetoresistance with respect to the magnetic field $(B)$ at different angles between $B$ and the electric current $(I)\left(\theta=0^{\circ}-90^{\circ}\right)$. The inset zooms in on the lower MR part, showing negative MR at $\theta=90^{\circ}$ (longitudinal negative MR), and it depicts the corresponding measurement configurations. (b) Magnetoresistance measured in different rotating angles around $\theta=90^{\circ}$ with the interval of every $0.2^{\circ}$. The negative MR appeared at a narrow region around $\theta=90^{\circ}$, and most obviously when $B / / I$. Either positive or negative deviations from $90^{\circ}$ would degenerate and ultimately kill the negative MR in the whole range of the magnetic field. Inset: The minima of MR curves at different angles $\left(88^{\circ}-92.2^{\circ}\right)$ in a magnetic field from 1 to $6 \mathrm{~T}$. (c) The negative MR at $\theta=90^{\circ}$ (open circles) and fitting curves (red dashed lines) at various temperatures. $T=1.8$, $10,25,50,75$, and $100 \mathrm{~K}$. (d) Magnetoresistance in the perpendicular magnetic field component, $B \times \cos \theta$. The misalignment indicates the $3 \mathrm{D}$ nature of the electronic states. 
regime [6,37]. Under the external magnetic field, the lowenergy states near the Weyl points reorganize to form Landau states for the motion perpendicular to the field and leave the momentum $k$ parallel to the field still a good quantum number. As shown in Fig. 1(d), the zeroth Landau levels are chiral, with the chirality determined by that of the Weyl point. With the additional electric field along the same direction, the equation of motion for the electrons on the chiral modes gives $\hbar(d k / d t)=-e E$, which adiabatically pumps electrons from one valley to another one with opposite chirality. In order to equilibrate the charge imbalance between the positive- and the negative-handed fermions resulting from the chiral anomaly, large-momentum internode scattering is required. However, in a sufficiently clean sample, such processes are considerably weak. Hence, the internode scattering time $\tau$ is very large, causing a remarkable increase in conductivity. Then, solving the corresponding Boltzmann equation under the semiclassical approximation gives chiral-anomaly-contributed conductivity as [6]

$$
\sigma^{\text {chiral }}=\frac{e^{2}}{4 \pi^{2} \hbar c} \frac{v}{c} \frac{(e B v)^{2}}{E_{F}^{2}} \tau,
$$

where $v$ is the Fermi velocity near the Weyl points and $E_{F}$ denotes the chemical potential measured from the energy of the Weyl points. The above chiral part of the conductivity increasing quadratically with magnetic field $B$ leads to negative MR, which has the maximum effect with $E$ parallel to $B$. Of course, the total conductivity of the system will also include other contributions from the nonchiral states as well, such as conventional conductivity of nonlinear band contributions, $\sigma_{\mathrm{N}}$, and the WAL, $\sigma_{\mathrm{WAL}}$, which may weaken the negative MR effect or even overwhelm it if the nonchiral part dominates the dc transport. Therefore, in order to observe the chiral negative MR, the high-quality sample with chemical potential close enough to the Weyl point is crucial. In this work, this can be roughly recognized by the coexistence of $\mathrm{SdH}$ oscillations and giant transverse MR [see Fig. 2(a)], which usually implies small Fermi surfaces around the Fermi level [38]. In addition, the misposition of the electric leads and/or the unavoidable misalignment of the magnetic field and the electric current may also strongly weaken the negative MR because of the huge positive MR and Hall resistivity signals in this material. The high precision of the measurement setup enables a very reliable measurement and manifests itself by the highly symmetrical original data of $\rho_{\mathrm{xx}}$ observed in opposite magnetic fields (see Ref. [39]).

Quantitative analyses are carried out by fitting the negative $\mathrm{MR}$ at $\theta=90^{\circ}$ with the semiclassical formula [26] in the magnetic field $-4 \mathrm{~T}<B<4 \mathrm{~T}$ :

$$
\sigma(B)=\left(1+C_{W} H^{2}\right) \cdot \sigma_{\mathrm{WAL}}+\sigma_{\mathrm{N}},
$$

$$
\sigma_{\mathrm{WAL}}=\sigma_{0}+a \sqrt{H}
$$

and

$$
\sigma_{N}^{-1}=\rho_{0}+A \cdot H^{2}
$$

$\sigma_{0}$ is the zero field conductivity, and $C_{W}$ is a positive parameter that originates from the topological $E \cdot B$. Such a topological term will generate a chiral current in the nonorthogonal magnetic and electric fields. $\sigma_{\mathrm{WAL}}$ and $\sigma_{\mathrm{N}}$ are those from the WAL effect and conventional nonlinear band contributions around the Fermi level, respectively. The fitting parameters are listed in Ref. [39]. Figure 2(c) shows the experimental data (open circles) and the fitting curves (red dashed lines) at various temperatures. The negative MR is suppressed by increasing the temperature, and it ultimately disappears at around $75 \mathrm{~K}$. Remarkably, the nicely fitted experimental data from Eq. (2) further confirmed the negative MR originating from the chiral anomaly. Near the zero field, the data show a sharp upturn, which is well fitted by including the $\sigma_{\mathrm{WAL}}$ term in Eq. (2), signaling that the WAL effect stemmed from the strong spin-orbit interactions.

We note that, for a magnetic field higher than $9 \mathrm{~T}$, the MR in Fig. 2(b) will change sign to be positive again. Since the separation between the Weyl points in $k$ space is about 3\%-8\% of the zone boundary [23], which is quite large compared with the energy scale of the Zeeman coupling, it is very unlikely that the Weyl points will annihilate in pairs in a high magnetic field. One possible explanation is the Coulomb interaction among the electrons occupying the chiral states. Since the degeneracy of the chiral states, as well as the density of states at the Fermi level, goes linearly with the magnetic field, with the increments of the magnetic field, the system behaves more and more like a one-dimensional system with large internal degeneracy, where the Coulomb interaction may play a dominating role and make the resistivity rise again with the field. Also, the negative MR is very likely to be smeared out by the component of transverse MR caused by the misalignment of the magnetic field with the current, as discussed above. The extremely narrow angle dependence of negative MR in Fig. 2(b) demonstrates this point of view.

Measurements are also implemented by tilting the magnetic field with respect to the (001) facet but keeping $B \perp I$ [see Fig. S1(b)]. As expected, no trace of negative MR has been detected, and this adds to the growing evidence that the negative MR originates from the chiral $E \cdot B$ term. Variations of MR as a function of the perpendicular component of the magnetic field, $B \cos \theta$, are studied and shown in Fig. 2(d). The misalignment of the curves indicates the 3D nature of the electronic structure in TaAs. This is reconfirmed by the explicit $\mathrm{SdH}$ oscillations in the whole angle range [see Fig. 2(a)]. 


\section{B. Temperature and magnetic field dependence of the Hall resistivity}

Figure 3(a) displays the magnetic field dependence of Hall resistivity $\rho_{\mathrm{xy}}$ in the temperature range from 2 to $300 \mathrm{~K}$. As shown in the left inset of Fig. 3(a), at low temperatures, the negative slope in high magnetic fields indicates that electrons mainly dominate the transport processes. And the Hall resistivity near $0 \mathrm{~T}$ presents nonlinear behavior. However, the Hall coefficient changes sign from negative to positive at higher temperatures [see the right inset of
Fig. 3(a)], implying that the electron-dominated conduction mechanism transforms to a hole-type mechanism. The transition temperature as shown in Fig. 3(b) is about $100 \mathrm{~K}$. At this temperature, remarkably, the nonlinear feature of $\rho_{\mathrm{xy}}$ extends to a high field, both the Hall resistivity and its slope change their signs, signaling the possibility of the coexistence of high-mobility electrons with lowmobility holes $[38,40,41]$. We identified this by fitting the experimental data of longitudinal conductivity $\sigma_{x x}$ and Hall conductivity $\sigma_{x y}$ with a two-carrier model [40,41]:
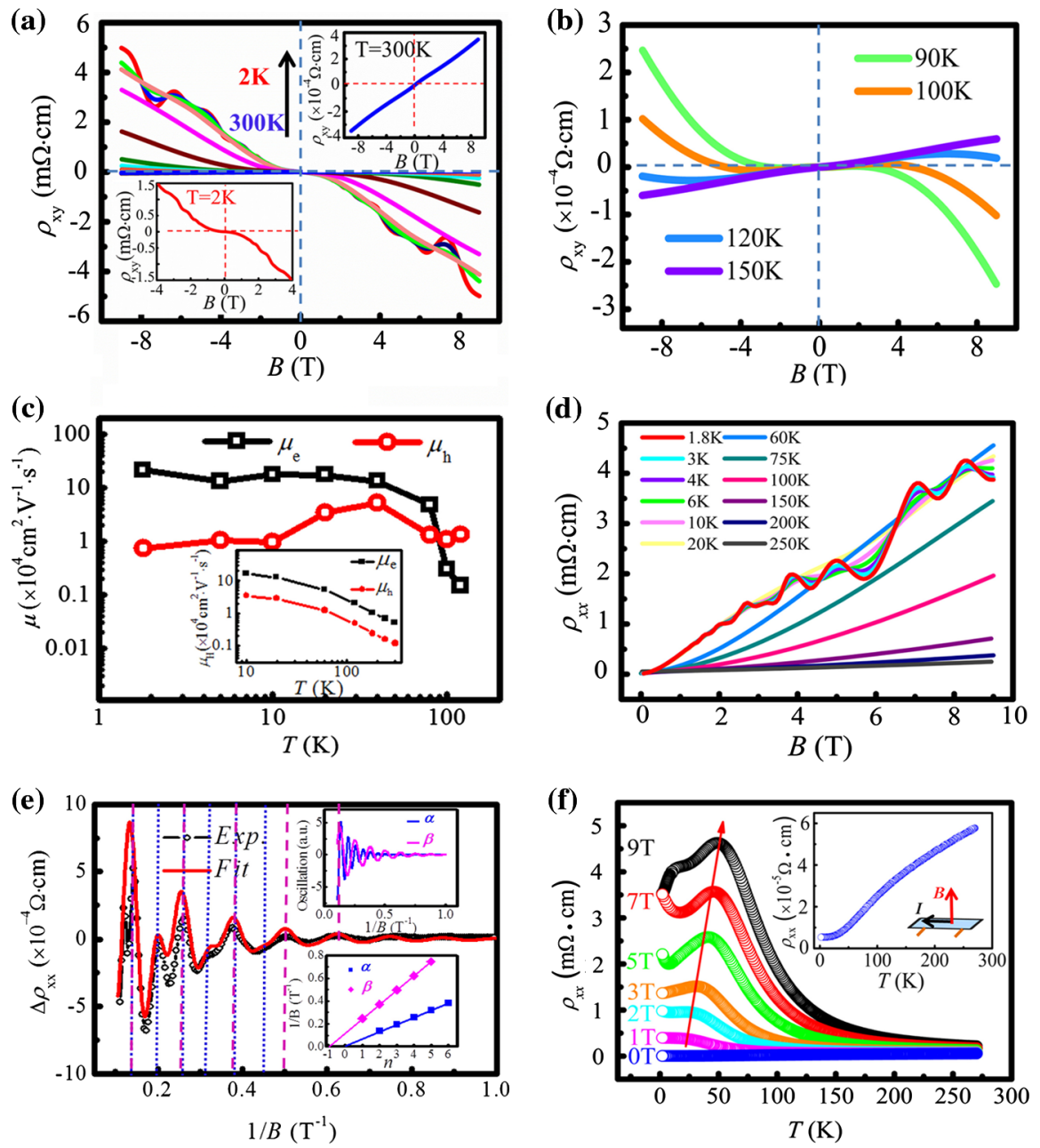

FIG. 3. Temperature dependence of Hall resistivity and resistivity for TaAs. (a) Hall resistivity measured at various temperatures from 2 to $300 \mathrm{~K}$. The lower-left and the upper-right insets show the Hall resistivity at 2 and $300 \mathrm{~K}$, respectively. The obvious SdH oscillation demonstrates the high quality of the sample. (b) Hall resistivity at $\mathrm{T}=90,100,120$, and $150 \mathrm{~K}$. (c) Temperature dependence of carrier mobility $\mu_{e}$ and $\mu_{h}$ of electrons and holes deduced by the two-carrier model. Main panel: Fitting with $\sigma_{x y}$; inset: fitting with $\sigma_{x x}$. (d) Magnetic field dependence of resistivity with $\theta=0^{\circ}$ at representative temperatures. (e) Main panel: Oscillatory components of $\rho_{\mathrm{xx}}$ at $1.8 \mathrm{~K}$, obtained by subtracting the $\rho_{\mathrm{xx}}$ at $20 \mathrm{~K}$. The open circles are the experimental data, and the red line is the best fit by two oscillatory frequency components. Upper inset: Two frequency components $\alpha=16 \mathrm{~T}$ and $\beta=7 \mathrm{~T}$ extracted from raw oscillation patterns in the main panel. Lower inset: Landau-level index plots $1 / B$ versus $n$ for different oscillation frequencies $\alpha=16 \mathrm{~T}$ and $\beta=7 \mathrm{~T}$. We assigned integer indices to the $\Delta \rho_{\mathrm{xx}}$ peak positions in $1 / B$ and indicated them with blue and pink dashed lines in the main panel. (f) The temperature dependence of resistivity in the magnetic field perpendicular to the electric current. The red arrow indicates that the resistivity peak moves to high temperatures under much higher magnetic fields. The inset gives the measurement configuration and zooms in on the case of $0 \mathrm{~T}$. 


$$
\begin{aligned}
\sigma_{x x} & =\frac{n_{e} q \mu_{e}}{1+\left(\mu_{e} B\right)^{2}}+\frac{\sigma_{x x}(0)-n_{e} q \mu_{e}}{1+\left(\mu_{h} B\right)^{2}} \\
\sigma_{x y} & =\left[n_{e} \mu_{e}^{2} \frac{1}{1+\left(\mu_{e} B\right)^{2}}-n_{h} \mu_{h}^{2} \frac{1}{1+\left(\mu_{h} B\right)^{2}}\right] e B .
\end{aligned}
$$

Here, $n_{e}$ (or $n_{h}$ ) is the carrier density of electrons (or holes), $\mu_{e}\left(\mu_{h}\right)$ is the mobility of electrons (holes), and $\sigma_{x x}(0)$ is the longitudinal conductivity at $0 \mathrm{~T}$. We list the fitting parameters in Ref. [39], Tables S2 and S3. The excellent agreement between the experimental data and the two-carrier model (see Fig. S2 in Ref. [39]) at different temperatures confirms the coexistence of electrons with holes in TaAs. The large conventional MR may be attributed to the compensation of the electron and hole pockets [42]. The mobility of two kinds of carriers at various temperatures is extracted and presented in Fig. 3(c). We find that the results estimated from $\sigma_{x x}$ (the main panel) and $\sigma_{x y}$ (the inset panel) are roughly in the same order of magnitude in a large temperature range, especially for $\mu_{e}$. In the low-temperature regime, the concentration of two kinds of carriers is comparable, while the mobility of electrons is much larger than that of holes. $\mu_{e}$ increases with decreasing temperature and reaches an extremely high value of $\mu_{e} \approx 1.8 \times 10^{5} \mathrm{~cm}^{2} \mathrm{~V}^{-1} \mathrm{~s}^{-1}$ at $T=10 \mathrm{~K}$. Above $80 \mathrm{~K}$, however, $\mu_{e}$ in the main panel starts to drop dramatically by almost 2 orders of magnitude, and $\mu_{h}$ has weak temperature dependence, suggesting that the holes dominate the transport properties at higher temperatures. This result is in agreement with the results derived from the Hall resistivity. Based on the above analyses, we can conclude that at low temperatures, the electrons with extremely high mobility are Weyl fermions, which dominate the negative MR behavior in the parallel electric and magnetic fields.

\section{Analysis of the SdH oscillations}

Magnetic field dependence of resistivity $\rho_{\mathrm{xx}}$ at various temperatures is measured at $\theta=0^{\circ}$ and shown in Fig. 3(d). Obviously, the quantum oscillations at lower temperatures are suppressed with increasing temperature and ultimately eliminated at around $20 \mathrm{~K}$. We extracted the quantum oscillations by subtracting the MR data at $20 \mathrm{~K}$ from those at $1.8 \mathrm{~K}$, as shown in Fig. 3(e). The oscillatory spectrum is considerably complex because of the contribution from several subbands, which is consistent with the bandstructure calculations. In order to get more reliable information on the oscillation phase factor, we try to fit the data using an expression for the quantum oscillations [43-45],

$$
\Delta \rho_{x x}=\rho(T, B) \cos [2 \pi(F / B-\gamma+\delta)] .
$$

Here, $F$ is the oscillation frequency, and $\gamma=\frac{1}{2}-\left(\varphi_{B} / 2 \pi\right)$ is the Onsager phase factor taking the value $\gamma=0,1$ (or $\gamma=1 / 2$ ) for the nontrivial (trivial) Berry phase $\varphi_{B}$. $\delta$ is a phase offset, with the value of $\delta=0$ (or $\pm \frac{1}{8}$ ) for the 2D (or 3D) system. Using Eq. (7), experimental data of $\Delta \rho_{\mathrm{xx}}$ are well fitted by two oscillatory frequency components $\alpha=16 \mathrm{~T}$ and $\beta=7 \mathrm{~T}$. The upper inset in Fig. 3(e) plots the two components extracted from raw oscillation patterns. We also estimated the values of $\gamma$ from Landan index plots [see the lower inset in Fig. 3(e)]. The linear extrapolations of the Landau level index $n$ versus $1 / B$ plot yield the values of $\gamma-\delta$ to be around zero (for $\alpha=16 \mathrm{~T}$ ) and $0.96(\beta=7 \mathrm{~T})$, respectively, which give strong evidence for the presence of nontrivial $\pi$ Berry phases arising from 3D Weyl electrons. Data of four additional samples from different batches are also analyzed to further confirm the existence of the nontrivial Berry phases in TaAs (see Ref. [39]). The cross-sectional area of the Fermi surface (FS) $A_{F}$ can be obtained according to the Onsager relation $F=\left(\varnothing_{0} / 2 \pi^{2}\right) A_{F}$, where $\varnothing_{0}$ is the flux quantum. The calculated values of $6.674 \times 10^{-4} \AA^{-2}$ and $15.26 \times$ $10^{-4} \AA^{-2}$ are only $1.998 \times 10^{-6} \%$ and $4.568 \times 10^{-6} \%$ of the cross-sectional area of the first BZ, respectively, further implying each FS is enclosing a Weyl point. We also note that $\rho_{\mathrm{xx}}(B)$ has a transition from quadratic field dependence at low fields to linear dependence at higher fields. The crossover field strength increases monotonically with the temperature, as introduced in more detail in Ref. [39] (see Fig. S3). In Fig. 3(f), the temperature dependence of resistivity $\rho_{\mathrm{xx}}$ at $\theta=0^{\circ}$ is plotted. In a zero magnetic field, TaAs exhibits a metallic behavior down to $1.8 \mathrm{~K}$ [see the inset of Fig. 3(f)]. The applied magnetic field not only significantly increases the resistivity, but it also stimulates a crossover from metalliclike to insulatorlike behavior, which may be related to the formation of the Landau levels under the magnetic field.

\section{Fermi surface and band structure}

The FS cross sections determined by the quantum oscillations are roughly consistent with the first-principle calculations, showing that there are three kinds of Fermi surfaces in the first BZ [Figs. 4(a) and 4(b)]. The two that are clearly visible are bananalike hole pockets and datelike electron pockets. The eight bananalike hole pockets are from band 2, as shown in Fig. 4(c). The 16 datelike electron pockets are enclosing the Weyl nodes in the $k_{z}=0.592 \pi$ plane (W1) [23]. These nodal points are around $21 \mathrm{meV}$ below the Fermi level. The band structures around W1 along $k_{x}, k_{y}$, and $k_{z}$ are shown in Figs. 4(d)-(f), indicating $\mathrm{W} 1$ is not strongly anisotropic, with averaged Fermi velocity along three directions: 2.025, 2.387, and $0.231 \mathrm{eV} \bullet \AA$, respectively. The Weyl node in the $k_{z}=0$ plane, W2, is around $2 \mathrm{meV}$ higher than the Fermi level, which contributes to the third type of Fermi surface, which is too tiny and is marked by dots in Fig. 4(b). W2 is strongly anisotropic, as shown by its band structures along $k_{x}, k_{y}$, 
(a)

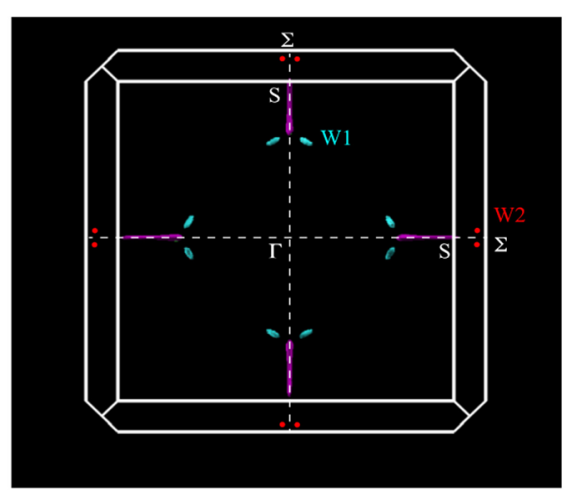

(c)

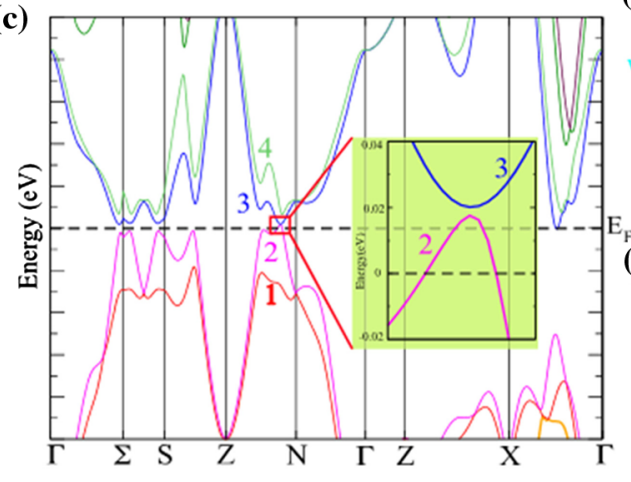

(b)

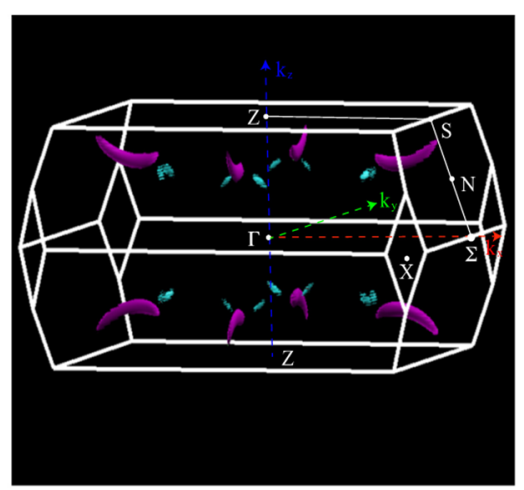

(d)

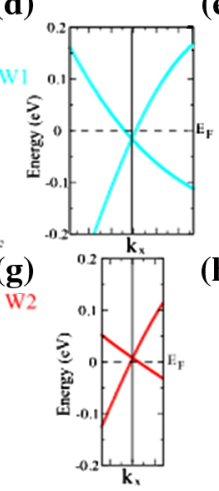

(e)

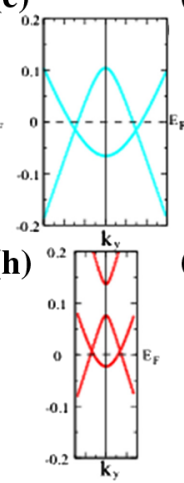

(f)

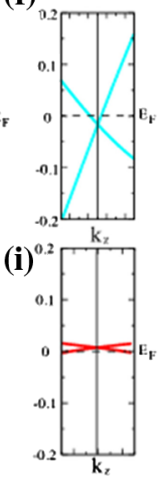

FIG. 4. Theoretical analyzing of Fermi surfaces in TaAs. (a,b) First-principles-calculated Fermi surfaces in the top view and side view, respectively. (c) The band structure with SOC included. (d-f) and (g-i) Band structure around Weyl nodes in the $k_{z}=0.592 \pi$ (W1) and $k_{z}=0$ (W2) planes along the $k_{x}, k_{y}$, and $k_{z}$ directions, respectively.

and $k_{z}$ in Figs. 4(g)-4(i). The Fermi velocities along them are $1.669,2.835$, and $0.273 \mathrm{eV} \AA$, respectively. Combining the information obtained from both quantum oscillation experiments and first-principle calculations, we can conclude that it is likely that the FSs associated with $16 \mathrm{~T}$ and 7 T oscillating frequencies enclose the Weyl points W1 and W2, respectively, and dominate the dc transport in TaAs, which, on the other hand, strengthens our conclusion that the negative MR observed in this material is truly due to the chiral anomaly.

\section{CONCLUSION}

In summary, the transport properties of proposed WSM TaAs have been studied in detail. Our results suggest that there are both $n$ and $p$ types of carriers in TaAs, which nearly compensate each other. Further analyses of the Hall effect data through two-carrier fittings obtained the electronic motilities at different temperatures and gave an unexpected high value of $\mu_{e} \approx 1.8 \times 10^{5} \mathrm{~cm}^{2} \mathrm{~V}^{-1} \mathrm{~s}^{-1}$ for the electrons at $10 \mathrm{~K}$. Giant linear MR as high as $80000 \%$ at $9 \mathrm{~T}$ has been found with a magnetic field perpendicular to the current, which is quite similar to the behavior in the Dirac semimetal $\mathrm{Cd}_{3} \mathrm{As}_{2}$. When the external magnetic field is rotated to be parallel with the current, the MR becomes negative between $1 \mathrm{~T}$ and $9 \mathrm{~T}$, with the most negative value around $-30 \%$. This unusual negative MR is the first evidence for the chiral anomaly associated with the Weyl points in TaAs. The $\pi$ Berry phase acquired by electrons in cyclotron orbits is also strong evidence of Weyl points. Thus, TaAs is a rare nonmagnetic compound confirmed by the experiments to host the WSM state, which has paved the way for more experimental studies in this completely new research territory.

\section{ACKNOWLEDGMENTS}

The authors wish to thank L. Lu, Z. P. Hou, L. Shan, C. H. Li, and C. Ren for their fruitful discussions and helpful comments. This work was supported by the National Basic Research of China Program 973 (Grants No. 2015CB921303, No. 2011CBA00108, and No. 2013CB921700), the "Strategic Priority Research Program (B)" of the Chinese Academy of Sciences (Grants No. XDB01020300 and No. XDB07020100) and the National Natural Science Foundation of China.

X. H. and L.Z. contributed equally to this work.

[1] Z. Fang, N. Nagaosa, K. S. Takahashi, A. Asamitsu, R. Mathieu, T. Ogasawara, H. Yamada, M. Kawasaki, Y. Tokura, and K. Terakura, The Anomalous Hall Effect and Magnetic Monopoles in Momentum Space, Science 302, 92 (2003). 
[2] H. Weyl, Elektron und Gravitation, Z. Phys. 56, 330 (1929).

[3] H. Nielsen and M. Ninomiya, Absence of Neutrinos on a Lattice: (I). Proof by Homotopy Theory, Nucl. Phys. B185, 20 (1981); Absence of Neutrinos on a Lattice: (II). Intuitive Topological Proof, Nucl. Phys. B193, 173 (1981).

[4] X. Wan, A. M. Turner, A. Vishwanath, and S. Y. Savrasov, Topological Semimetal and Fermi-Arc Surface States in the Electronic Structure of Pyrochlore Iridates, Phys. Rev. B 83, 205101 (2011).

[5] G. Xu, H. M. Weng, Z. Wang, X. Dai, and Z. Fang, Chern Semimetal and the Quantized Anomalous Hall Effect in $\mathrm{HgCr}_{2} \mathrm{Se}_{4}$, Phys. Rev. Lett. 107, 186806 (2011).

[6] D. T. Son and B. Z. Spivak, Chiral Anomaly and Classical Negative Magnetoresistance of Weyl Metals, Phys. Rev. B 88, 104412 (2013).

[7] S. M. Young, S. Zaheer, J. C. Y. Teo, C. L. Kane, E. J. Mele, and A. M. Rappe, Dirac Semimetal in Three Dimensions, Phys. Rev. Lett. 108, 140405 (2012).

[8] Z. J. Wang, Y. Sun, X. Q. Chen, C. Franchini, G. Xu, H. M. Weng, X. Dai, and Z. Fang, Dirac Semimetal and Topological Phase Transitions in $\mathrm{A}_{3} \mathrm{Bi}(\mathrm{A}=\mathrm{Na}, \mathrm{K}, \mathrm{Rb})$, Phys. Rev. B 85, 195320 (2012).

[9] Z. J. Wang, H. M. Weng, Q. S. Wu, X. Dai, and Z. Fang, Three-Dimensional Dirac Semimetal and Quantum Transport in $\mathrm{Cd}_{3} \mathrm{As}_{2}$, Phys. Rev. B 88, 125427 (2013).

[10] B. J. Yang and N. Nagaosa, Classification of Stable Three-Dimensional Dirac Semimetals with Nontrivial Topology, Nat. Commun. 5, 4898 (2014).

[11] H. B. Nielsen and M. Ninomiya, The Adler-Bell-Jackiw Anomaly and Weyl Fermions in a Crystal, Phys. Lett. 130B, 389 (1983).

[12] S. A. Parameswaran, T. Grover, D. A. Abanin, D. A. Pesin, and A. Vishwanath, Probing the Chiral Anomaly with Nonlocal Transport in Three-Dimensional Topological Semimetals, Phys. Rev. X 4, 031035 (2014).

[13] K. Fukushima, D. Kharzeev, and H. Warringa, Chiral Magnetic Effect, Phys. Rev. D 78, 074033 (2008).

[14] A. C. Potter, I. Kimchi, and A. Vishwanath, Quantum Oscillations from Surface Fermi Arcs in Weyl and Dirac Semimetals, Nat. Commun. 5, 5161 (2014).

[15] Z. K. Liu, B. Zhou, Y. Zhang, Z. J. Wang, H. M. Weng, D. Prabhakaran, S. K. Mo, Z. X. Shen, Z. Fang, X. Dai, Z. Hussain, and Y. L. Chen, Discovery of a Three-Dimensional Topological Dirac Semimetal, $\mathrm{Na}_{3} \mathrm{Bi}$, Science 343, 864 (2014).

[16] M. Neupane, S. Y. Xu, R. Sankar, N. Alidoust, G. Bian, C. Liu, I. Belopolski, T. R. Chang, H. T. Jeng, H. Lin, A. Bansil, F. C. Chou, and M. Z. Hasan, Observation of a Three-Dimensional Topological Dirac Semimetal Phase in High-Mobility $\mathrm{Cd}_{3} \mathrm{As}_{2}$, Nat. Commun. 5, 3786 (2014).

[17] Z. K. Liu, J. Jiang, B. Zhou, Z. J. Wang, Y. Zhang, H. M. Weng, D. Prabhakaran, S.-K. Mo, H. Peng, P. Dudin, T. Kim, M. Hoesch, Z. Fang, X. Dai, Z. X. Shen, D. L. Feng, Z. Hussain, and Y.L. Chen, A Stable ThreeDimensional Topological Dirac Semimetal $\mathrm{Cd}_{3} \mathrm{As}_{2}$, Nat. Mater. 13, 677 (2014).

[18] S. Jeon, B. B. Zhou, A. Gyenis, B. E. Feldman, I. Kimchi, A. C. Potter, Q. D. Gibson, R. J. Cava, A. Vishwanath, and A. Yazdani, Landau Quantization and Quasiparticle
Interference in the Three-Dimensional Dirac Semimetal $\mathrm{Cd}_{3} \mathrm{As}_{2}$, Nat. Mater. 13, 851 (2014).

[19] S. Y. Xu, C. Liu, S. K. Kushwaha, R. Sankar, J. W. Krizan, I. Belopolski, M. Neupane, G. Bian, N. Alidoust, T.-R. Chang et al., Observation of Fermi Arc Surface States in a Topological Metal, Science 347, 294 (2015).

[20] A. A. Burkov and L. Balents, Weyl Semimetal in a Topological Insulator Multilayer, Phys. Rev. Lett. 107, 127205 (2011).

[21] G. B. Halasz and L. Balents, Time-Reversal Invariant Realization of the Weyl Semimetal Phase, Phys. Rev. B 85, 035103 (2012).

[22] J. P. Liu and D. Vanderbilt, Weyl Semimetals from Noncentrosymmetric Topological Insulators, Phys. Rev. B 90, 155316 (2014).

[23] H. M. Weng, C. Fang, Z. Fang, B. A. Bernevig, and X. Dai, Weyl Semimetal Phase in Noncentrosymmetric TransitionMetal Monophosphides, Phys. Rev. X 5, 011029 (2015).

[24] S. M. Huang, S. Y. Xu, I. Belopolski, C. C. Lee, G. Q. Chang, B. K. Wang, N. Alidoust, G. Bian, M. Neupane, A. Bansil, H. Lin, and M. Z. Hasan, A Weyl Fermion Semimetal with Surface Fermi Arcs in the Transition Metal Monopnictide TaAs Class, Nat. Commun. 6, 7373 (2015).

[25] P. Hosur and X. L. Qi, Recent Developments in Transport Phenomena in Weyl Semimetals, C.R. Phys. 14, 857 (2013).

[26] H. J. Kim, K. S. Kim, J. F. Wang, M. Sasaki, N. Satoh, A. Ohnishi, M. Kitaura, M. Yang, and L. Li, Dirac versus Weyl Fermions in Topological Insulators: Adler-Bell-Jackiw Anomaly in Transport Phenomena, Phys. Rev. Lett. 111, 246603 (2013).

[27] B. Q. Lv, H. M. Weng, B. B. Fu, X. P. Wang, H. Miao, J. Ma, P. Richard, X. C. Huang, L. X. Zhao, G. F. Chen, Z. Fang, X. Dai, T. Qian, and H. Ding, Experimental Discovery of Weyl Semimetal TaAs, Phys. Rev. X 5, 031013 (2015).

[28] B. Q. Lv, N. Xu, H. M. Weng, J. Z. Ma, P. Richard, X. C. Huang, L. X. Zhao, G. F. Chen, C. E. Matt, F. Bisti, V. N. Strokov, J. Mesot, Z. Fang, X. Dai, T. Qian, M. Shi, and H. Ding, Observation of Weyl Nodes in TaAs, Nat. Phys., doi:10.1038/nphys3426 (2015).

[29] S. Y. Xu, I. Belopolski, N. Alidoust, M. Neupane, G. Bian, C. L. Zhang, R. Sankar, G. Q. Chang, Z. J. Yuan, C. C. Lee, S. M. Huang, H. Zheng, J. Ma, D. S. Sanchez, B. K. Wang, A. Bansil, F. C. Chou, P. P. Shibayev, H. Lin, S. Jia, and M. Z. Hasan, Discovery of a Weyl Fermion Semimetal and Topological Fermi Arcs, Science, doi:10.1126/science .aaa9297 (2015).

[30] L. Lu, Z. Y. Wang, D. X. Ye, L.X. Ran, L. Fu, J. D. Joannopoulos, and M. Soljačić, Experimental Observation of Weyl Points, Science, doi:10.1126/science.aaa9273 (2015).

[31] C. L. Zhang, Z. J. Yuan, S. Y. Xu, Z. Q. Lin, B. B. Tong, M.Z. Hasan, J. F. Wang, C. Zhang, and S. Jia, Tantalum Monoarsenide: An Exotic Compensated Semimetal, arXiv:1502.00251.

[32] S. Furuseth, K. Selte, and A. Kjekshus, On the Arsenides and Antimonodes of Tantalum, Acta Chem. Scand. 19, 95 (1965).

[33] http://www.openmx-square.org.

[34] J. P. Perdew, K. Burke, and M. Ernzerhof, Generalized Gradient Approximation Made Simple, Phys. Rev. Lett. 77, 3865 (1996). 
[35] J. Xiong, S. K. Kushwaha, T. Liang, J. W. Krizan, W. D. Wang, R. J. Cava, and N. P. Ong, Signature of the Chiral Anomaly in a Dirac Semimetal: A Current Plume Steered by a Magnetic Field, arXiv:1503.08179.

[36] C. Shekhar, F. Arnold, S. C. Wu, Y. Sun, M. Schmidt, N. Kumar, A. G. Grushin, J. H. Bardarson, R. D. dos Reis, M. Naumann, M. Baenitz, H. Borrmann, M. Nicklas, E. Hassinger, C. Felser, and B. H. Yan, Large and Unsaturated Negative Magnetoresistance Induced by the Chiral Anomaly in the Weyl Semimetal TaP, arXiv:1506.06577.

[37] E. V. Gorbar, V. A. Miransky, and I. A. Shovkovy, Chiral Anomaly, Dimensional Reduction, and Magnetoresistivity of Weyl and Dirac Semimetals, Phys. Rev. B 89, 085126 (2014).

[38] S. Ishiwata, Y. Shiomi, J. S. Lee, M. S. Bahramy, T. Suzuki, M. Uchida, R. Arita, Y. Taguchi, and Y. Tokura, Extremely High Electron Mobility in Aphonon-Glass Semimetal, Nat. Mater. 12, 512 (2013).

[39] See Supplemental Material at http://link.aps.org/ supplemental/10.1103/PhysRevX.5.031023 for the original magnetoresistance data at different angles, fitting of the Hall conductivity with two-carrier model, magnetoresistance at lower magnetic field range and the $\mathrm{SdH}$ oscillations of four samples from different batches.

[40] H. Takahashi, R. Okazaki, Y. Yasui, and I. Terasaki, Low-Temperature Magnetotransport of the Narrow-Gap Semiconductor FeSb ${ }_{2}$, Phys. Rev. B 84, 205215 (2011).

[41] B. Xia, P. Ren, A. Sulaev, P. Liu, S. Q. Shen, and L. Wang, Indications of Surface-Dominated Transport in Single Crystalline Nanoflake Devices of Topological Insulator $\mathrm{Bi}_{1.5} \mathrm{Sb}_{0.5} \mathrm{Te}_{1.8} \mathrm{Se}_{1.2}$, Phys. Rev. B 87, 085442 (2013).

[42] M. N. Ali, J. Xiong, S. Flynn, J. Tao, Q. D. Gibson, L. M. Schoop, T. Liang, N. Haldolaarachchige, M. Hirschberger, N. P. Ong, and R. J. Cava, Large, Non-Saturating Magnetoresistance in $\mathrm{WTe}_{2}$, Nature (London) 514, 205 (2014).

[43] G. P. Mikitik and Yu. V. Sharlai, Manifestation of Berry's Phase in Metal Physics, Phys. Rev. Lett. 82, 2147 (1999).

[44] H. Murakawa, M. S. Bahramy, M. Tokunaga, Y. Kohama, C. Bell, Y. Kaneko, N. Nagaosa, H. Y. Hwang, and Y. Tokura, Detection of Berry's Phase in a Bulk Rashba Semiconductor, Science 342, 1490 (2013).

[45] E. N. Adams and T.D. Holstein, Quantum Theory of Transverse Galvano-Magnetic Phenomena, J. Phys. Chem. Solids 10, 254 (1959). 